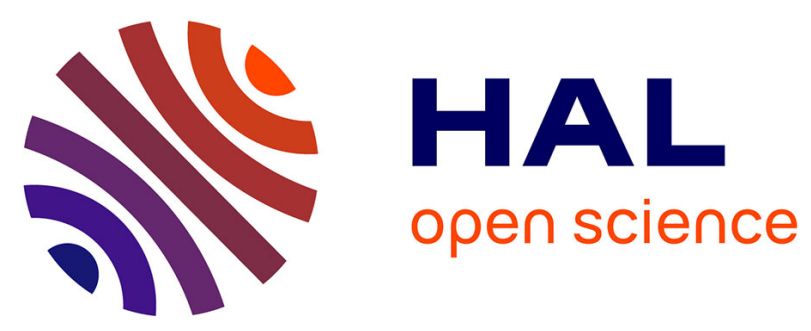

\title{
Etude par analyse de Fourier des phénomènes non linéaires affectant la propagation ultrasonore dans un fluide. L'émission paramétrique. L'autodémodulation non linéaire
}

P. Alais, P.-Y. Hennion, Pierre Cervenka

\section{To cite this version:}

P. Alais, P.-Y. Hennion, Pierre Cervenka. Etude par analyse de Fourier des phénomènes non linéaires affectant la propagation ultrasonore dans un fluide. L'émission paramétrique. L'autodémodulation non linéaire. Revue de Physique Appliquée, 1985, 20 (6), pp.285-297. 10.1051/rphysap:01985002006028500 . jpa-00245335

\section{HAL Id: jpa-00245335 \\ https://hal.science/jpa-00245335}

Submitted on 1 Jan 1985

HAL is a multi-disciplinary open access archive for the deposit and dissemination of scientific research documents, whether they are published or not. The documents may come from teaching and research institutions in France or abroad, or from public or private research centers.
L'archive ouverte pluridisciplinaire HAL, est destinée au dépôt et à la diffusion de documents scientifiques de niveau recherche, publiés ou non, émanant des établissements d'enseignement et de recherche français ou étrangers, des laboratoires publics ou privés. 


\title{
REVUE DE PHYSIQUE APPLIQUÉE
}

\section{Etude par analyse de Fourier des phénomènes non linéaires affectant la propagation ultrasonore dans un fluide. L'émission paramétrique. L'autodémodulation non linéaire}

\author{
P. Alais, P.-Y. Hennion et P. Cervenka \\ Université Paris VI, Laboratoire de Mécanique Physique (*), 2, place de la Gare de Ceinture, \\ 78210 St Cyr l'Ecole, France
}

(Reçu le 29 novembre 1984, accepté le 4 février 1985)

\begin{abstract}
Résumé. - On étudie la propagation non linéaire de faisceaux acoustiques dans les fluides en utilisant l'analyse de Fourier spatiale. Les modes plans atténués classiques ne conviennent plus et doivent être remplacés par des modes plans « inhomogènes" se propageant dans toutes les directions, mais dont les plans équiamplitudes sont parallèles au plan de référence.

Le traitement complet de l'interaction non linéaire faible entre deux faisceaux primaires harmoniques, définis par leur spectre angulaire, est développé aussi bien en champ proche que lointain, avec peu d'approximations. Ce formalisme est ensuite adapté au cas de l'émission par un transducteur plan d'un signal harmonique modulé en amplitude.

Ces résultats sont étendus au cas des amplitudes finies pour lequel l'extra-atténuation non linéaire affecte les ondes primaires. On donne des résultats numériques et expérimentaux.
\end{abstract}

\begin{abstract}
The nonlinear propagation of acoustical beams in fluids is studied using a spatial Fourier analysis. The classical plane attenuated modes are not convenient and must be replaced by " inhomogeneous " plane modes propagating in any direction but with equi-amphitude planes parallel to the reference plane.

The full treatment of the weak interaction between two primary harmonic beams, defined by their angular spectrum, is given as well for the nearfield as for the farfield, with very few approximations. This formalism is then adapted to the problem of a plane transducer driven with an amplitude modulated carrier.

These results are extended to the finite amplitude case in which primary waves are affected by non linear attenuation. Numerical and experimental results are given.
\end{abstract}

\section{Introduction.}

On sait que la théorie de la propagation linéaire acoustique résulte d'une approximation négligeant d'une part la convection de l'onde acoustique par le mouvement du milieu matériel où elle se propage, d'autre part les non-linéarités résultant du comportement thermodynamique du milieu. En fait ces deux phénomènes sont à l'origine d'interactions non linéaires à tout niveau de puissance, dont l'importance pourrait souvent devenir considérable, si elle n'était le plus souvent gommée par l'atténuation linéaire qui affecte les ondes acoustiques dans les liquides

(*) UA 868 du C.N.R.S. proportionnellement au carré de leur fréquence. Bien que, on le verra plus loin, la production de fréquences plus élevées (sommation de fréquences) soit plus efficace en transfert énergétique, les ondes ainsi créées sont le plus souvent atténuées rapidement par le milieu et ne sont à l'origine que d'une distorsion plus ou moins forte pouvant aller jusqu'à conférer à l'onde le profil bien connu de dents de scie. Il n'en va pas de même des ondes de basse fréquence créées par différence de fréquences car ces ondes, très faiblement atténuées, se propagent plus loin que les ondes créées initialement et finalement subsistent seules au loin. Le point de départ de cet article concerne la structure de ce rayonnement résiduel de basse fréquence, dans le cas où l'énergie extraite du rayonne- 
ment initial par les phénomènes non linéaires ne l'affecte pas sensiblement en regard de l'atténuation linéaire normale. Cette situation est celle communément dénommée interaction non linéaire faible. Comme il n'y a plus dans ce cas d'effet en retour de l'interaction non linéaire, on peut retenir un modèle simple où le rayonnement primaire, décrit par le potentiel des vitesses $\Phi^{\mathbf{I}}$, vérifie l'équation de propagation linéaire et le rayonnement secondaire, décrit par le potentiel des vitesses $\Phi^{\text {II }}$, vérifie la même équation des ondes affecté d'un terme de sources résultant de la connaissance du champ primaire :

$$
\Phi^{\mathrm{I}}=0, \quad \square^{\prime} \equiv\left(1+\frac{\eta}{\rho_{0} C_{0}^{2}} \frac{\partial}{\partial t}\right) \nabla^{2}-\frac{1}{C_{0}^{2}} \frac{\partial}{\partial t^{2}}
$$

$$
\begin{aligned}
\Phi^{\mathrm{II}} & =S^{\mathrm{I}} \\
S^{\mathrm{I}} & =\frac{1}{C_{0}^{2}} \frac{\partial}{\partial t}\left[\left(\nabla \Phi^{\mathrm{I}}\right)^{2}+\frac{B}{2 A} \frac{1}{C_{0}^{2}}\left(\frac{\partial \Phi^{\mathrm{I}}}{\partial t}\right)^{2}\right]
\end{aligned}
$$

où $\rho_{0}$ et $C_{0}$ représentent respectivement la masse volumique et la célérité acoustique du fluide et $\eta$ un terme d'atténuation s'identifiant classiquement pour un liquide à $\eta=\frac{4}{3} \eta^{\prime}+\eta^{\prime \prime}$ où $\eta^{\prime}$ et $\eta^{\prime \prime}$ sont les viscosités de cisaillement et de volume du liquide. Ces équations résultent d'un développement asymptotique classique des équations de l'hydrodynamique [1].

L'étude des phénomènes non linéaires faibles suivant un tel modèle a été proposée dès 1957 par Westervelt [2] avec un formalisme équivalent à celui des équations (1) et (2) et une solution sous forme intégrale pour cette dernière. En suggérant la possibilité de l'émission paramétrique [3], Westervelt a été à l'origine de nombreux travaux tant théoriques qu'expérimentaux visant à préciser le fonctionnement du sonar paramétrique lequel n'est autre qu'un sonar haute fréquence excité par deux fréquences comprises dans la bande passante du dispositif. Le rayonnement utile est créé à la fréquence différence (de battements) par les sources virtuelles induites par le terme $S^{\mathbb{I}}$ de l'équation (2), et se propage beaucoup plus loin que le rayonnement primaire dont il est issu, avec des propriétés de directivité tout à fait remarquables dues au fait que l'étendue du réseau de sources virtuelles ainsi créé est considérable et limitée dans le sens de la propagation seulement par la longueur d'atténuation du rayonnement primaire. Le modèle simple de Westervelt, où on suppose que le rayonnement primaire est très directif, concentre le réseau des sources sur l'axe de tir et permet d'accéder par une intégrale simple au résultat classique pour la directivité en amplitude :

$$
f(\theta)=\left(1+\pi^{2} v^{2} \theta^{4} / 4 \alpha_{0}^{2} C_{0}^{2}\right)^{-1 / 2},
$$

où $v$ et $\alpha_{0}$ représentent la fréquence et l'atténuation non linéaire. Ce résultat qui peut troubler le physicien habitué à manier des réseaux orientés dans un plan quasi perpendiculaire à l'axe d'émission et non pas selon l'axe d'émission (" end fire array " est l'expression anglaise imagée du phénomène) est lié à la nondispersion acoustique et au fait que la construction cohérente d'une onde acoustique par interaction non linéaire ne peut se faire que selon la direction commune de deux ondes acoustiques colinéaires, ce pour satisfaire à la sommation (ou différence) simultanée des fréquences et des vecteurs d'ondes. Naturellement dans la pratique la directivité effective du transducteur intervient et la plupart des travaux entrepris ont visé à compléter le modèle de Westervelt pour en tenir compte, et en déduire des méthodes approchées de calcul pour les différentes configurations rencontrées d'interaction en champ proche ou en champ lointain (Berktay et Leahy [4], Mellen et Moffett $[5,6])\left({ }^{1}\right)$.

Après que soit rappelée au § 2 la possibilité d'adapter l'analyse de Fourier à la propagation linéaire en milieu absorbant, l'objet du §3 est de montrer comment on peut accéder de façon correcte, au moins pour les faibles niveaux, à la directivité de l'émission paramétrique, à partir de ce formalisme commode et jusqu'ici peu employé par les acousticiens [8].

A l'évidence le modèle d'analyse des phénomènes non linéaires reposant sur les équations (1) et (2) retenu pour sa simplicité ne peut rendre compte des régimes de niveaux forts pour lesquels une partie importante de l'énergie primaire rayonnée est transférée à des fréquences harmoniques rapidement atténuées. C'est le phénomène bien connu de saturation qui limite la puissance rayonnée émise en raison de l'« extra-atténuation » non linéaire ainsi créée quand on cherche à élever le niveau de puissance d'excitation électrique $[9,10]$. Pour un sonar paramétrique, il en résulte une dégradation de la conversion basse fréquence due à la réduction apparente de la longueur d'atténuation du rayonnement primaire et une perte de directivité. Cependant l'essentiel des propriétés remarquables des émetteurs paramétriques concernant la directivité et la bande passante du rayonnement émis peut être décrit par le modèle proposé par les équations (1) et (2), et on verra au $\S 5$ comment prolonger la validité de ce modèle aux niveaux forts de puissance souvent atteints dans les applications.

\section{Le rayonnement primaire.}

Cette étude rappelle les caractéristiques déjà énoncées, [11], de la propagation acoustique linéaire dans un fluide absorbant dictée par l'équation (1). L'analyse de Fourier classique de la propagation linéaire sans atténuation peut être étendue sans difficulté au cas où l'atténuation doit être prise en compte. On sait que la théorie classique fournit une expression d'un

( ${ }^{1}$ ) Pour une bibliographie détaillée, voir [7] 
rayonnement acoustique harmonique de pulsation $\omega=2 \pi v$ se propageant selon la direction moyenne $\mathrm{O} z$ sous la forme spectrale :

$$
\left.\begin{array}{l}
\Phi(\mathbf{r}, t)=\int A_{0}(\mathbf{f}) \mathrm{e}^{j(\mathbf{k r}-\omega t)} \mathrm{df}, \\
\mathbf{k}=\frac{\omega}{C_{0}} \mathbf{n}=2 \pi \mathbf{f}+k_{\mathbf{z}} \mathbf{z},
\end{array}\right\}
$$

à savoir une décomposition en modes plans décrits à partir du vecteur d'onde $\mathbf{k}$ satisfaisant l'équation de dispersion du fluide $k=\omega / C_{0}$. Le spectre $A(\mathbf{f})$ n'est autre que la transformée de Fourier dans le plan $\Pi_{0}$, $(\mathbf{r}=\mathrm{Oz}+\mathbf{m})$, de l'amplitude complexe de $\Phi$ dans ce plan :

$$
\begin{aligned}
\Phi_{0}(\mathbf{m}, t) & =U_{0}(\mathbf{m}) \mathrm{e}^{-j \omega t} \\
U_{0}(\mathbf{m}) & =\int A_{0}(\mathbf{f}) \mathrm{e}^{j 2 \pi \mathbf{f} . \mathbf{m}} \mathrm{df} .
\end{aligned}
$$

On sait que l'avantage essentiel de la répartition spectrale est de fournir très simplement l'évolution du rayonnement au cours de la propagation selon $\mathrm{O} z$ à savoir l'expression de l'amplitude complexe de $\Phi$ dans le plan $\Pi_{z},(\mathbf{r}=z \mathbf{z}+\mathbf{m}, z=\mathrm{Cte})$ :

$$
\begin{gathered}
U_{z}(\mathbf{m})=\int A_{0}(\mathbf{f}) \cdot \mathrm{e}^{j k . \mathrm{r}} \mathrm{df}=\int A_{z}(\mathbf{f}) \mathrm{e}^{j 2 \pi f \cdot m} \mathrm{df}, \\
A_{z}(\mathbf{f})=A_{0}(\mathbf{f}) H_{\mathrm{Oz}}(\mathbf{f}) ; \quad H_{\mathrm{Oz}}(\mathbf{f})=\mathrm{e}^{j k_{z} z}, \\
U_{z}(\mathbf{m})=U_{0}(\mathbf{m}) * h_{\mathrm{Oz}}(\mathbf{m}), \quad h_{\mathrm{Oz}}(\mathbf{m})=\text { T.F. }^{-1}\left(H_{\mathrm{O}_{z}}\right),
\end{gathered}
$$

où l'expression (7) représente une formulation particulière de la relation de Rayleigh Sommerfeld [12].

Toutes les expressions précédentes s'étendent sans difficulté au cas d'un fluide avec atténuation à la condition de substituer dans (3) au mode plan classique non atténué $\exp [j(\mathbf{k} . \mathbf{r}-\omega t)]$, de signature $\exp (j 2 \pi \mathbf{f} . \mathbf{m})$ dans le plan $\Pi_{0}$ un mode de même signature vérifiant l'équation de propagation (1). Ceci impose l'utilisation d'un vecteur d'onde $\mathbf{k}^{\prime}$ de composante réelle $2 \pi \mathrm{f}$ dans $\Pi_{0}$ et vérifiant l'équation de dispersion associée à (1) :

$$
k^{\prime 2}\left(C_{0}^{2}-j \omega \eta / \rho_{0}\right)=\omega^{2} .
$$

La composante $k_{z}^{\prime}$ est alors complexe : $k_{z}^{\prime}=k_{z}(\mathbf{f})+$ $j \alpha_{z}(\mathbf{f})$ et la partie imaginaire représente une atténuation exclusive en $z$ du mode plan.

Dans le cas, seul d'intérêt pratique, où la longueur d'atténuation reste grande devant la longueur d'onde $\left(\omega \eta / \rho_{0} C_{0}^{2} \ll 1\right)$, un calcul élémentaire montre que la partie réelle du vecteur d'onde $\mathbf{k}^{\prime}$ associé à la fréquence spatiale $\mathbf{f}$ dans $\Pi_{0}$ s'identifie au vecteur $\mathbf{k}$ obtenu dans le cas sans atténuation et qu'on peut écrire très valablement :

$$
\mathbf{k}^{\prime} \cong \mathbf{k}+j(\alpha / \cos \theta) \mathbf{z}, \quad \alpha=\frac{\omega^{2} \eta}{2 \rho_{0} C_{0}^{3}}, \quad \theta=(\mathbf{z}, \mathbf{k}),
$$

où $\alpha$ représente l'atténuation des modes plans atténués ordinaires. La représentation spectrale (3) peut alors être étendue à l'expression nouvelle faisant appel à ces nouveaux modes plans atténués exclusivement en $z$ :

$$
\Phi(\mathbf{r}, t)=\int A_{0}(\mathbf{f}) \mathrm{e}^{-\alpha_{z} z} \mathrm{e}^{j k_{z} z} \mathrm{e}^{j 2 \pi(\mathbf{f . r}-v t)} \mathrm{df},
$$

où

$$
\alpha_{z}(\mathbf{f})=\alpha / \cos \theta, \quad \theta(\mathbf{f})=\operatorname{Arcsin}\left(\frac{f C_{0}}{v}\right) .
$$

Les formules (5), (6) et (7) sont alors utilisables en introduisant en lieu et place des opérateurs $H_{\mathbf{O}_{2}}$ et $h_{\mathrm{Oz}}$ les nouveaux opérateurs :

$$
\left.\begin{array}{ll}
H_{\mathrm{O} z}^{\prime}=H_{\mathrm{Oz}} A_{\mathrm{O} z}, & A_{\mathrm{Oz}}(\mathrm{f})=\mathrm{e}^{-\alpha z / \cos \theta} \\
h_{\mathrm{O} z}^{\prime}=h_{\mathrm{Oz}} * a_{\mathrm{O} z}, & a_{\mathrm{Oz}}(\mathrm{m})=\text { T.F. } \\
-1 & \left(A_{\mathrm{O} z}\right) .
\end{array}\right\}
$$

Dans tous les cas où l'approximation paraxiale classique de Fresnel est utilisable pour $k_{z}$ on peut alors étendre cette approximation à la propagation atténuée par les relations

$$
\left.\begin{array}{l}
k_{z} \cong \frac{2 \pi}{\lambda}-\pi \lambda f^{2}, \quad \theta \cong \lambda f, \\
\alpha_{z} \cong \alpha\left(1+\frac{\lambda^{2} f^{2}}{2}\right), \quad \lambda=\frac{C_{0}}{v},
\end{array}\right\}
$$

ce qui permet de formuler les approximations de Fresnel des opérateurs $H_{\mathrm{O}_{z}}^{\prime}(\mathbf{f})$ et $h_{\mathrm{O}_{z}}^{\prime}(\mathbf{m})$ selon :

$$
\begin{aligned}
& H_{\mathrm{O} z}^{\prime}=H_{\mathrm{Oz}} A_{\mathrm{O} z},\left\{\begin{array}{l}
H_{\mathrm{O} z} \cong \mathrm{e}^{j \frac{2 \pi}{\lambda} z} \mathrm{e}^{-j \pi \lambda z f^{2}} \\
A_{\mathrm{O} z} \cong \mathrm{e}^{-\alpha z} \mathrm{e}^{-\frac{\alpha \lambda^{2} f^{2}}{2} z},
\end{array}\right. \\
& h_{\mathrm{O} z}^{\prime}=h_{\mathrm{O} z} * a_{\mathrm{O} z},\left\{\begin{array}{l}
h_{\mathrm{O} z} \cong \mathrm{e}^{j \frac{2 \pi}{\lambda} z} \frac{\mathrm{e}^{j \frac{m^{2}}{\lambda z}}}{j \lambda z}, \\
a_{\mathrm{O} z} \cong \frac{2 \pi}{\alpha z \lambda^{2}} \mathrm{e}^{-\alpha z} \mathrm{e}^{-\frac{2 \pi^{2}}{\alpha \lambda^{2} z} m^{2}}
\end{array}\right.
\end{aligned}
$$

L'expression (7) étendue au cas absorbant permet alors de constater que l'effet de l'atténuation sur un rayonnement harmonique décrit par l'amplitude $U_{0}(\mathrm{~m})$ dans le plan initial $\Pi_{0}$ se traduit au niveau plan $\Pi_{z}$ par l'expression

$$
U_{z}^{\prime}(\mathbf{m})=U_{z}(\mathbf{m}) * a_{\mathrm{Oz}}(\mathbf{m}),
$$

déduite de l'expression $U_{z}$ obtenue sans atténuation, par une convolution par une gaussienne de largeur caractéristique $\delta=\lambda \sqrt{\frac{\alpha z}{2 \pi}}$ ayant pour effet, outre l'atténuation globale en $\mathrm{e}^{-\alpha z}$, de lisser les fréquences spatiales élevées et d'augmenter légèrement la largeur de la tache focale pour un dispositif focalisant, tout en apportant un effet bénéfique d'apodisation. Ces effets particuliers n'ont d'ailleurs d'importance réelle que pour des atténuations importantes liées à de 
grandes ouvertures angulaires ce qui est le cas en microscopie acoustique.

Naturellement toutes les considérations précédentes s'étendent aux rayonnements non harmoniques à la condition d'introduire un spectre spatio-temporel représentatif du rayonnement émis dans le plan initial $\Pi_{0}$ soit :

$$
\Phi(\mathbf{m}, t)=\int A_{0}(\mathbf{f}, v) \mathrm{e}^{j 2 \pi(\mathbf{f . r}-v t)} \mathrm{d} \mathbf{f} d v
$$

Dans tout plan $\Pi_{z}$ le rayonnement peut être décrit par un spectre $A_{z}(\mathbf{f}, v)$ déduit de $A_{0}$ à toute fréquence $v$ par les formules précédentes. Par ailleurs la formulation de Fourier qui vient d'être donnée de la propagation linéaire avec atténuation est bien adaptée à la description des phénomènes d'interactions non linéaires qui vont être étudiés plus loin.

\section{Le rayonnement secondaire : interaction paramé- trique de deux rayonnements primaires harmoniques.}

Cette partie est consacrée exclusivement à l'interaction non linéaire résultant de la propagation de deux rayonnements primaires harmoniques émis aux fréquences $v_{1}$ et $v_{2}$ décrits respectivement dans le plan initial $\Pi_{0}$ par les spectres $A_{1}(\mathbf{f})$ et $A_{2}(\mathbf{f})$. Chacun d'eux est donc fourni par l'expression

$$
\Phi_{i}^{\mathrm{I}}(\mathbf{r}, t)=\int A_{i}(\mathbf{f}) \mathrm{e}^{-\alpha_{i z} z} \mathrm{e}^{j k_{i z} z} \mathrm{e}^{j 2 \pi\left(\mathbf{f r}-v_{i} t\right)} \mathrm{df}, \quad i=1,2 .
$$

Le rayonnement secondaire est créé par les termes de sources quadratiques $S^{\mathbf{I}}$ donné par l'équation (2) à partir des potentiels $\Phi_{i}$. Ces termes quadratiques sont bien sûr à l'origine de l'addition et de la différence des fréquences $v_{i}$ et des phases $\varphi_{i}$ selon la relation

$$
\cos \varphi_{i} \cos \varphi_{j}=\frac{1}{2}\left[\cos \left(\varphi_{i}+\varphi_{j}\right)+\cos \left(\varphi_{i}-\varphi_{j}\right)\right],
$$

et sont responsables de quatre rayonnements harmoniques émis aux fréquences $2 v_{1}, 2 v_{2}, v_{+}=v_{1}+v_{2}$, $v_{-}=v_{2}-v_{1}$. Nous ne retiendrons par la suite que le terme de fréquence $v_{-}$car le rayonnement correspondant subsiste seul au loin. Cependant les autres rayonnements peuvent être déterminés par des formules tout à fait similaires.

L'utilisation d'un langage complexe reste évidemment souhaitable pour le rayonnement secondaire comme pour le rayonnement primaire et on montre simplement que le terme de sources à la fréquence $v_{-}$ est la partie réelle d'un terme en $\exp \left\{-j 2 \pi v_{-} t\right\}$ proportionnel à $\Phi_{1}^{*} \Phi_{2}$ et la linéarité de ce terme relative à $\Phi_{1}^{*}$ et à $\Phi_{2}$ permet d'atteindre un résultat pour le potentiel $\Phi^{\text {II }}$ de la forme :

$$
\Phi^{\mathrm{II}}(\mathbf{r}, t)=\iint A_{1}^{*}\left(\mathbf{f}_{1}\right) A_{2}\left(\mathbf{f}_{2}\right) \mathcal{J}\left(\mathbf{f}_{1}, \mathbf{f}_{2}, \mathbf{r}, t\right) \mathrm{df_{1 }} \mathrm{df}_{2},
$$

où le noyau $\partial$ représente le résultat à la fréquence $v_{-}$ de l'interaction non linéaire des modes plans atténués élémentaires $\left(v_{1}, \mathbf{f}_{1}\right)$ et $\left(v_{2}, \mathbf{f}_{2}\right)$ auxquels fait appel l'analyse de Fourier décrite en $\$ 2$. La détermination du noyau $j$ est donc le problème élémentaire à résoudre d'abord, et on verra que le résultat conduit à une représentation spectrale naturelle de $\Phi^{\text {II }}$.

3. 1 INTERACTION NON LINÉAIRE DE DEUX MODES PLANS ATTÉNUÉS EN $z$ [13]. - Les rayonnements considérés s'expriment simplement selon :

$$
\begin{gathered}
\Phi_{i}^{\mathrm{I}}(\mathbf{r}, t)=A_{i} \mathrm{e}^{-\alpha_{i z} z} \mathrm{e}^{j \varphi_{i}}, \\
\varphi_{i}=2 \pi\left(\mathbf{f}_{i} \cdot \mathbf{m}-v_{i} t\right)+k_{i z} z, \quad(i=1,2) \quad(20) \\
\alpha_{i z}=\frac{\alpha_{i}}{\cos \theta_{i}}, \quad \alpha_{i}=\frac{\eta \omega_{i}^{2}}{2 \rho_{0} C_{0}^{3}}, \quad \theta_{i}=\operatorname{Arcsin}\left(\frac{C_{0} f_{i}}{v_{i}}\right), \\
k_{i z}=2 \pi \frac{v_{i}}{C_{0}} \cos \theta_{i} .
\end{gathered}
$$

Le terme de source à la fréquence $v_{-}=v_{2}-v_{1}$ s'écrit compte tenu de l'expression (2) de $S^{\mathrm{I}}$ et tenant compte de ce que

$$
\nabla \Phi_{i}^{\mathbf{I}}=j \mathbf{k}_{i}^{\prime} \Phi_{i} \cong j \mathbf{k}_{i} \Phi_{i}
$$

dans la mesure où $\alpha_{i} \ll k_{i}$ :

$$
\begin{aligned}
S_{-}^{\mathrm{I}} \cong-j \frac{\omega_{-}}{C_{0}^{2}} A_{1}^{*} A_{2} \exp \left[-\left(\frac{\alpha_{1}}{\cos \theta_{1}}+\frac{\alpha_{2}}{\cos \theta_{2}}\right) z\right] \times \\
\quad \times\left\{\left(-j \mathbf{k}_{1}\right)\left(j \mathbf{k}_{2}\right)+\frac{B}{2 A} \frac{1}{C_{0}^{2}}\left(j \omega_{1}\right)\left(-j \omega_{2}\right)\right\} \mathrm{e}^{j\left[\left(\mathbf{k}_{2}-\mathbf{k}_{1}\right) \cdot \mathbf{r}-\omega_{-} t\right]}
\end{aligned}
$$

Ce terme a des variations de phase temporelles et spatiales en $x$ et $y$ identiques à la phase $\varphi_{-}$du mode plan atténué défini par les fréquences temporelle $v_{-}$ et spatiale $\mathbf{f}_{-}=\mathbf{f}_{2}-\mathbf{f}_{1}$, soit

$$
\varphi_{-}=2 \pi\left(\mathbf{f}_{-} \cdot \mathbf{m}-v_{-} t\right)+k_{-z} z \text {. }
$$

La solution attendue pour le rayonnement $\Phi^{\text {II }}$ doit donc posséder la même strúcture spatio-temporelle en $(x y t)$, ne comporter qu'une variation non triviale en $z$ et pouvoir s'écrire sous la forme du mode atténué $\left(v_{-}, f_{-}\right)$modulé en $z$ :

$$
\Phi_{\text {II }}=A(z) \mathrm{e}^{-\frac{\alpha_{-}}{\cos \theta_{-}} z} \mathrm{e}^{j \varphi_{-}}
$$


On sait que la création du rayonnement secondaire $\Phi^{\text {II }}$ est parfaitement constructive si l'écart de phase :

$$
\Delta \varphi=\varphi_{2}-\varphi_{1}-\varphi_{-}=\left(k_{2 z}-k_{1 z}-k_{-z}\right) z=\Delta k z
$$

disparaît, ce qui en l'absence de dispersion acoustique se produit seulement pour l'interaction de modes colinéaires $\left(\mathbf{k}_{1} / / \mathbf{k}_{2}\right)$. Dans le cas général l'injection d'une solution du type (23) dans l'équation (2) dotée du terme de source (22) conduit à l'équation différentielle

$$
\begin{aligned}
\left(1-j \frac{\omega_{-} \eta}{\rho_{0} C_{0}^{2}}\right)\left[\frac{\mathrm{d}^{2} A_{-}}{\mathrm{d} z^{2}}\right. & \left.+2 j\left(k_{-z}+j \alpha_{-z}\right) \frac{\mathrm{d} A_{-}}{\mathrm{d} z}\right] \mathrm{e}^{-\alpha_{-z} z} \mathrm{e}^{j \varphi_{-}}= \\
= & -j \frac{\omega_{-}}{C_{0}^{2}} A_{1}^{*} A_{2}\left[\mathbf{k}_{1} \cdot \mathbf{k}_{2}+\frac{\omega_{1} \omega_{2}}{C_{0}^{2}} \frac{B}{2 A}\right] \mathrm{e}^{-\left(\alpha_{1 z}+\alpha_{2 z}\right) z} \mathrm{e}^{j \varphi_{-}^{\prime}}, \quad\left(\varphi_{-}^{\prime}=\varphi_{2}-\varphi_{1}\right) .
\end{aligned}
$$

Comme le rayonnement basse fréquence a une longueur d'atténuation encore plus grande devant sa longueur d'onde on peut supposer que $\omega_{-} \eta / \rho_{0} C_{0}^{2} \ll 1$ et $\alpha_{-z} \ll k_{-z}$, et de plus effectuer l'approximation parabolique qui revient à supposer que $\left|\frac{\mathrm{d}^{2} A_{-}}{\mathrm{d} z^{2}}\right| \ll\left|j k_{-z} \frac{\mathrm{d} A_{-}}{\mathrm{d} z}\right|$; ce qui réduit l'équaton (25) à :

$$
\begin{aligned}
\frac{\mathrm{d} A_{-}}{\mathrm{d} z} & =-\frac{A_{1}^{*} A_{2}}{2 C_{0} \cos \theta}\left(\mathbf{k}_{1} \cdot \mathbf{k}_{2}+\frac{B}{2 A} \frac{\omega_{1} \omega_{2}}{C_{0}^{2}}\right) \mathrm{e}^{-v z}, \text { avec } v=\alpha-j \Delta k, \\
\alpha & =\frac{\alpha_{1}}{\cos \theta_{1}}+\frac{\alpha_{2}}{\cos \theta_{2}}-\frac{\alpha_{-}}{\cos \theta_{-}}>0, \quad \Delta k=k_{2 z}-k_{1 z}-k_{-z},
\end{aligned}
$$

dont l'intégration immédiate donne :

$$
A_{-}(z)=\int_{0}^{z} \frac{\mathrm{d} A_{-}}{\mathrm{d} z} \mathrm{~d} z=A_{1}^{*} A_{2} I\left(\mathbf{f}_{1}, \mathbf{f}_{2}, z\right), \quad \text { avec } \quad I=-\frac{1-\mathrm{e}^{-v z}}{2 C_{0} v \cos \theta_{-}}\left[\mathbf{k}_{1} \cdot \mathbf{k}_{2}+\frac{B}{2 A} \frac{\omega_{1} \omega_{2}}{C_{0}^{2}}\right] .
$$

Le rayonnement à l'infini, c'est-à-dire dans la région où les primaires sont devenues négligeables, peut être exprimé à partir du noyau constant :

$$
I_{\infty}=-\frac{1}{2 C_{0} v \cos \theta_{-}}\left[\mathbf{k}_{1} \cdot \mathbf{k}_{2}+\frac{B}{2 A} \frac{\omega_{1} \omega_{2}}{C_{0}^{2}}\right] .
$$

En approximation paraxiale $\left(\theta_{1}, \theta_{2} \ll 1\right)$, on obtient sans difficulté [14] une expression simplifiée du noyau $I$ :

$$
\begin{aligned}
& I=-\frac{2 \pi^{2} \beta}{C_{0}^{3}} v_{1} v_{2} \frac{1-\mathrm{e}^{-v z}}{v}, \quad \beta=1+B / 2 A, \\
& v=\alpha_{1}+\alpha_{2}-j \pi C_{0} \frac{v_{1} v_{2}}{v_{-}}\left(\frac{\mathrm{f}_{2}}{v_{2}}-\frac{\mathrm{f}_{1}}{v_{1}}\right)^{2} .
\end{aligned}
$$

L'interaction la plus constructive correspondant toujours à l'annulation de la partie imaginaire du terme $v$ c'est-à-dire à des modes colinéaires $\left(\mathbf{k}_{2} / / \mathbf{k}_{1}\right)$.

3.2 Formulation SPeCtrale du RAYONNEMENT SECONDAire ParamétriQue $\Phi^{\mathrm{II}}$ [15]. - Le calcul précédent a permis de préciser la nature du noyau $\mathcal{g}$ de l'intégrale (19)

où

$$
\begin{gathered}
J\left(\mathbf{f}_{1}, \mathbf{f}_{2}, \mathbf{r}, t\right)=I\left(\mathbf{f}_{1}, \mathbf{f}_{2}, z\right) \mathrm{e}^{-\alpha_{-z} z} \mathrm{e}^{j \varphi-}, \\
\varphi_{-}=2 \pi\left(\mathbf{f}_{-} . \mathbf{r}-v_{-} t\right)+k_{-z} z, \quad \mathbf{f}_{-}=\mathbf{f}_{2}-\mathbf{f}_{1}, \\
\alpha_{-}=\frac{\eta \omega^{2}}{2 \rho_{0} C_{0}^{3}}, \quad \alpha_{-z}=\alpha_{-}\left(1-\frac{C_{0}^{2} f_{-}^{2}}{v_{-}^{2}}\right)^{1 / 2} .
\end{gathered}
$$

On peut alors réécrire l'intégrale (19) sous la forme

$$
\Phi^{\mathrm{II}}(\mathbf{r}, t)=\int \mathrm{d} \mathbf{f}_{-} \int \mathrm{d} \mathbf{f}_{1} A_{1}^{*}\left(\mathbf{f}_{1}\right) A_{2}\left(\mathbf{f}_{1}+\mathbf{f}_{-}\right) I\left(\mathbf{f}_{1}, \mathbf{f}_{1}+\mathbf{f}_{-}, z\right) \mathrm{e}^{-\alpha_{-} z} \mathrm{e}^{j \varphi_{-}},
$$


ce qui est l'expression du rayonnement $\Phi^{\mathrm{II}}$ selon l'analyse spectrale décrite en $\S 2$ en associant à la représentation du rayonnement $\Phi^{\mathrm{II}}$ dans chaque plan $\Pi_{z}$ une décomposition spectrale en termes de modes plans atténués :

$$
\Phi^{\mathrm{II}}(\mathbf{r}, t)=\int A_{-}\left(\mathbf{f}_{-}, z\right) \mathrm{e}^{-\alpha_{-} z} \mathrm{e}^{j \varphi-} \mathrm{df} \mathbf{f}_{-},
$$

où le spectre $A_{-}\left(\mathbf{f}_{-}, z\right)$ s'exprime compte tenu de (19) selon :

$$
A_{-}\left(\mathbf{f}_{-}, z\right)=\int A_{1}^{*}\left(\mathbf{f}_{1}\right) A_{2}\left(\mathbf{f}_{1}+\mathbf{f}_{-}\right) I\left(\mathbf{f}_{1}, \mathbf{f}_{1}+\mathbf{f}_{-}, z\right) \mathrm{d} \mathbf{f}_{1}
$$

3.3 EMISSION PARAMÉTRIQUE D’UN PISTON PLAN [14]. - Il est intéressant d'illustrer les résultats précédents par le cas pratique d'un sonar paramétrique constitué d'un transducteur à face plane circulaire excité en mode d'épaisseur aux fréquences $v_{1}$ et $v_{2}$ comprises dans la bande passante. Si on admet en première approximation que les oscillations de la face émettrice conservent la planéité de celle-ci, les spectres des rayonnements primaires s'expriment aisément. La vitesse normale imposée dans le plan d'émission $\Pi_{0}$ s'écrit pour chaque fréquence $v_{i}$ :

$$
V_{i}(\mathbf{m}, t)=V_{i 0} P(\mathbf{m}) \mathrm{e}^{-j 2 \pi v_{i} t}=\frac{\partial}{\partial z} \Phi_{i}^{\mathrm{I}}(\mathbf{m}, t),
$$

où $\mathfrak{T}(\mathbf{m})$ est la fonction pupille de l'émetteur, et notant $\mathcal{A}(\mathbf{f})$ la Transformée de Fourier de la fonction pupille, la relation (34) s'écrit par transformée de Fourier des amplitudes complexes :

$$
V_{i 0} \cdot \mathcal{A}(\mathbf{f})=j k_{i z} A_{i}(\mathbf{f}) .
$$

Si on considère le cas seul intéressant en pratique d'un transducteur directif pour les deux fréquences concernées on peut écrire $k_{i z} \cong \omega_{i} / C_{0}$ et donc les spectres primaires :

$$
A_{i}(\mathbf{f}) \cong \frac{C_{0} V_{i 0}}{j \omega_{i}} \mathcal{A}(\mathbf{f}) \cong \frac{P_{i}}{j \rho_{0} \omega_{i}} \mathcal{A}(\mathbf{f})
$$

notant $P_{i}=\rho_{0} C_{0} V_{i 0}$ l'amplitude de la pression primaire à la fréquence $v_{i}$ approximativement induite au niveau du transducteur.

Compte tenu des relations (33) et (30) le rayonnement paramétrique obtenu à la fréquence $v_{-}=v_{2}-v_{1}$ au-delà du domaine du rayonnement primaire s'exprime à partir du spectre :

$$
\begin{gathered}
A_{-}\left(\mathbf{f}_{-}\right)=-\frac{P_{1}^{*} P_{2} \beta}{2 \rho_{0} C_{0}^{3} \alpha} \int \mathrm{df}_{1} \mathcal{A}^{*}\left(\mathbf{f}_{1}\right) \mathcal{A}\left(\mathbf{f}_{1}+\mathbf{f}_{-}\right) \times \\
\times \frac{1}{1-j \gamma\left(\mathbf{f}_{1}-n \mathbf{f}_{-}\right)^{2}} \\
\text { où } n=\frac{v_{1}}{v_{-}}, \quad \beta=1+\frac{B}{2 A}, \quad \alpha=\alpha_{1}+\alpha_{2}-\alpha_{-}, \\
\gamma=\frac{\pi C_{0} v_{-}}{\alpha v_{1} v_{2}} .
\end{gathered}
$$

Dans la zone de Fraunhofer du rayonnement secondaire, le rayonnement lointain s'écrit alors directement à partir du spectre $\mathrm{A}_{-}$dans la direction $\boldsymbol{\theta}=\frac{C_{0} \mathbf{f}_{-}}{v_{-}}$

$$
\Phi_{-}(z, \theta) \cong \frac{1}{j \lambda z} A_{-}\left(\frac{\theta}{\lambda}\right) \mathrm{e}^{-\alpha_{-} z} \mathrm{e}^{j \varphi_{-}},
$$

où $\lambda=C_{0} / \nu_{-}$est la longueur d'onde du rayonnement secondaire auquel correspond l'amplitude de pression

où

$$
P(z, \boldsymbol{\theta})=-\frac{\pi \beta}{\rho_{0} C_{0}^{4} \alpha z} v_{-}^{2} \mathrm{e}^{-\alpha_{-} z} S(\boldsymbol{\theta}),
$$

$$
\begin{array}{r}
S(\boldsymbol{\theta})=\int \mathrm{df}_{1} \mathcal{A}^{*}\left(\mathbf{f}_{1}\right) \mathcal{A}\left(\mathbf{f}_{1}+\mathbf{f}_{-}\right) \frac{1}{1-j^{\gamma}\left(\mathbf{f}_{1}-n \mathbf{f}_{-}\right)^{2}} \\
\mathbf{f}_{-}=\frac{v_{-}}{C_{0}} \boldsymbol{\theta}
\end{array}
$$

3.4 EXEMPLES D'APPLiCATIONS. - Voici maintenant, sur un cas typique de l'émission paramétrique, les résultats obtenus [7] par les méthodes qu'on vient de détailler (Fig. 1). Il s'agit du champ lointain d'un piston de $16 \mathrm{~cm}$ de rayon fonctionnant dans l'eau de mer à une fréquence primaire moyenne de $150 \mathrm{kHz}$ et une fréquence secondaire de $10 \mathrm{kHz}$. Ces résultats sont présentés en échelle logarithmique conjointement à ceux obtenus en reprenant les calculs de Westervelt dans les mêmes conditions (tirets).

Dans le cas où la structure de l'émission primaire est plus complexe, et même pour la connaissance du champ non linéaire proche, ces méthodes conduisent encore assez commodément au résultat : ceci est illustré par le calcul de l'émission de transducteurs focalisants sphériques .[16] et cylindriques [17].

\section{Démodulation non linéaire de l'émission d'un piston plan.}

Cette étude constitue une nouvelle étape dans laquelle on s'intéresse à l'évolution non linéaire, toujours dañs les limites de puissances définies au $\S \mathbf{1}$, de l'émission d'un transducteur plan excité non plus selon deux fréquences discrètes mais selon un spectre temporel 


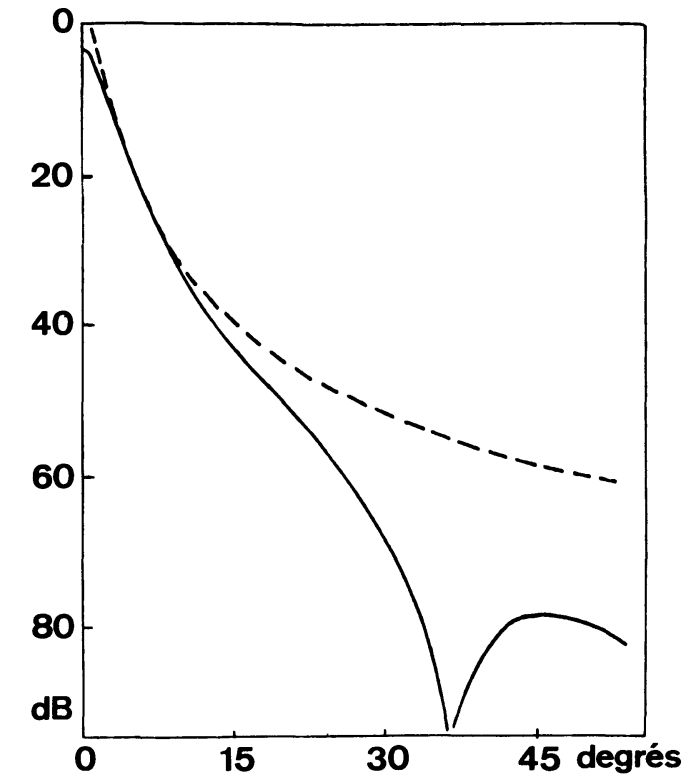

Fig. 1. - Diagramme de rayonnement secondaire d'un piston circulaire, en champ lointain, dans l'eau de mer. Rayon : $16 \mathrm{~cm}$, fréquence médiane $\mathrm{HF}: 150 \mathrm{kHz}$, fréquence paramétrique : $10 \mathrm{kHz}$ (— notre calcul ; - - - Westervelt).

[Farfield secondary beam pattern of a circular piston source, in sea water. Radius : $16 \mathrm{~cm}$; middle high frequency : $150 \mathrm{kHz}$; parametric frequency : $10 \mathrm{kHz}$.]

à bande étroite obtenu par modulation d'une fréquence porteuse $v_{0}$ égale à la fréquence centrale du transducteur.

Ce problème a été envisagé initialement par Berktay [18] dans le cadre du modèle de Westervelt, c'est-à-dire en considérant un faisceau d'ondes planes collimatées. Blackstock [19] a proposé une solution en transformant l'équation de propagation dans un fluide visqueux en l'équation de propagation de la chaleur. Les premières expériences ont concerné la propagation de trains d'ondes sinusoïdales [20-23]. En pratique, ce phénomène d'autodémodulation est pris en compte dans l'optimisation de la forme du signal enveloppe des sonars paramétriques en vue d'augmenter l'efficacité de la conversion [24]. Mais le sujet reste encore largement inexploré, et des recherches sont entreprises, en particulier sur la dépendance spatiale du signal paramétrique [25], et les effets de saturation.

4. 1 THÉORIE. - On examine maintenant ces problèmes en utilisant l'analyse de Fourier [26], avec les notations introduites au $\S 3$. Ecrivons la vitesse normale induite dans le plan $\Pi_{0}$ de la face parlante :

$$
V_{z}(\mathbf{m}, t)=\mathfrak{T}(\mathbf{m}) V_{0}(t) \mathrm{e}^{-j 2 \pi v_{0} t},
$$

où $\mathcal{T}(\mathrm{m})$ et $V_{0}(t)$ sont la fonction pupille du transducteur et la modulation de la vitesse induite à la fréquence $v_{0}$ de la face parlante. Le spectre spatio- temporel de la vitesse normale s'écrit alors notant $Y(v)$ la Transformée de Fourier de la fonction de modulation $V_{0}$ :

$$
A_{V_{z}}(\mathbf{f}, v)=\mathcal{A}(\mathbf{f}) \quad r\left(v-v_{0}\right) .
$$

Le spectre du faisceau primaire est alors défini comme en (36) par

$$
A(\mathbf{f}, v)=\frac{1}{j k_{z}} A_{V_{z}} \cong \frac{C_{0}}{j 2 \pi v} \mathcal{A}(\mathbf{f}) \quad r\left(v-v_{0}\right) .
$$

Le rayonnement primaire émis s'écrit :

$$
\begin{aligned}
\Phi(\mathbf{r}, t) & =\int \frac{C_{0} \mathrm{~d} v}{j 2 \pi v} \mathrm{r}\left(v-v_{0}\right) \times \\
& \times\left[\int \mathrm{df} \mathcal{A}(\mathbf{f}) \mathrm{e}^{-\frac{\alpha}{\cos \theta} z} \mathrm{e}^{j \boldsymbol{k}_{z} z} \mathrm{e}^{j 2 \pi(\mathbf{f} . \mathbf{m}-v t)}\right] .
\end{aligned}
$$

L'autodémodulation résulte de l'interaction paramétrique des fréquences contenues dans le spectre $r\left(v-v_{0}\right)$. Le champ acoustique basse fréquence ainsi créé est évidemment relié à la fonction de modulation $V_{0}(t)$ au travers d'un filtre spatio-temporel en général difficile à évaluer mais que l'étude précédente du § 3 permet d'atteindre assez facilement dans le cas particulier considéré ici d'un transducteur piston pour lequel les variables d'espace et de temps sont séparées dans l'expression (41) de la vitesse normale induite. Cette séparation des variables se retrouve pour les fréquences spatiale et temporelle dans l'expression (43) du spectre primaire et permet d'écrire à partir de l'expression (33) du spectre secondaire retenant pour l'écriture du noyau $I$ d'interaction la formule approchée (30) dans le champ lointain

$$
\begin{gathered}
{\left[\left(\alpha_{1}+\alpha_{2}\right) z \sim 2 \alpha_{0} z \gg 1\right]} \\
A_{-}(\mathbf{f}, v)=\left[\int \frac{\beta}{4 \alpha_{0} C_{0}} \Upsilon^{*}\left(v_{1}-v_{0}\right) \Upsilon\left(v_{1}+v-v_{0}\right) \mathrm{d} v_{1}\right] \times \\
\times\left[\int \frac{\mathcal{A}^{*}\left(\mathbf{f}_{1}\right) \mathcal{A}\left(\mathbf{f}_{1}+\mathbf{f}_{-}\right)}{1-j \pi \gamma\left(\mathbf{f}_{1}-n \mathbf{f}_{-}\right)^{2}} \mathrm{~d} \mathbf{f}_{1}\right]
\end{gathered}
$$

où l'étroitesse relative du spectre primaire émis $r\left(v-v_{0}\right)$ permet de ne retenir pour $n$ et $\gamma$ que les expressions approchées indépendantes de $v_{1}$

$$
\begin{gathered}
n=\frac{v_{1}}{v} \cong \frac{v_{0}}{v}, \\
\gamma=C_{0} \frac{v}{\left(\alpha_{1}+\alpha_{2}\right) v_{1}\left(\dot{v}_{1}+v\right)} \cong \frac{C_{0} v}{2 \alpha_{0} v_{0}^{2}} .
\end{gathered}
$$

Dans ces conditions le spectre secondaire conserve une séparabilité partielle des fréquences temporelle $v$ et spatiale $f$ soit :

$$
A_{-}(\mathbf{f}, v)=G(v) . S\left(v, \theta=C_{0} \mathbf{f} / v\right),
$$

où $G(v)$, si on se restreint à une modulation exclusive d'amplitude c'est-à-dire si la fonction $V_{0}(t)$ est réelle, 
s'identifie au produit d'autoconvolution du spectre $r(v)$ soit :

$$
G(v) \stackrel{\text { T.F. }}{\rightleftarrows} \frac{\beta}{4 C_{0} \alpha_{0}} V_{0}^{2}(t) .
$$

Par ailleurs la fonction $S(v, \theta)$ s'identifie évidemment à la directivité fournie en (40) du même transducteur utilisé en sonar paramétrique avec la réduction de fréquence $n=v_{0} / v_{-}$.

Dans la zone de Fraunhofer du rayonnement secondaire c'est-à-dire dans le champ lointain ce rayonnement peut s'exprimer par le champ de pression :

$$
P_{-}(z, \boldsymbol{\theta}, t)=\frac{\beta P_{0}}{8 \pi \alpha_{0} C_{0}^{2} z} \frac{\mathrm{d}^{2}}{\mathrm{~d} t^{2}}\left\{V_{0}^{2}(t)\right\} * \Sigma(t, \boldsymbol{\theta}),
$$

où $\Sigma(t, \boldsymbol{\theta})$ est la transformée inverse de Fourier temporelle $(v \rightleftarrows t)$ de la fonction $S(v, \theta)$.

On peut remarquer que sur l'axe $(\boldsymbol{\theta}=0, \mathbf{f}=0) \mathrm{si}$ on peut négliger $\gamma f_{1}^{2} \ll 1$ pour l'essentiel du rayonnement primaire émis ce qui est le cas des transducteurs assez directifs, la fonction $S(v, 0)$ s'identifie [14] à l'aire $S_{0}$ du transducteur et l'expression (47) se réduit à la formule :

$$
P_{-}(z, \mathbf{0}, t)=\frac{\beta P_{0} S_{0}}{8 \pi \alpha_{0} C_{0}^{2} z} \frac{\mathrm{d}^{2}}{\mathrm{~d} t^{2}}\left\{V_{0}^{2}(t)\right\},
$$

proposée par Berktay [18] à partir du modèle simplifié de Westervelt. On voit ici que l'autodémodulation non linéaire conduit à la dérivée seconde du signal de modulation détecté quadratiquement.

Par ailleurs on sait que le modèle de Westervelt fournit une fonction de directivité bien approchée par les transducteurs directifs laquelle s'écrit dans la formulation adoptée

$$
S(v, \boldsymbol{\theta}) \cong S_{0}\left(1+\pi^{2} v^{2} \theta^{4} / 4 C_{0}^{2} \alpha_{0}^{2}\right)^{-1 / 2} .
$$

4.2 RÉSUlTATS EXPÉRIMENTAUX. - Nous avons entamé au laboratoire [26] une étude expérimentale faisant appel à la configuration décrite à la figure (2). Une mémoire numérique et un capteur numérique de transitoires pilotés par un ordinateur et synchronisés sur une horloge digitale à la fréquence initiale du transducteur émetteur permettent d'émettre des signaux avec une fonction de modulation déterminée par l'ordinateur et de recevoir les signaux résultant de l'autodémodulation par l'intermédiaire d'une sonde électrostatique à large bande. La numérisation de ces derniers permet d'effectuer à l'aide de l'ordinateur une moyenne sur un nombre important de tirs ultrasonores et d'améliorer considérablement le rapport signal sur bruit. La sonde électrostatique a une réponse plate jusqu'à $1 \mathrm{MHz}$ et le transducteur émetteur centré sur $6,2 \mathrm{MHz}$ a une bande à $3 \mathrm{~dB}$ de plus de 1 mégahertz. Il se comporte comme un piston plan de $15 \mathrm{~mm}$ de diamètre avec une grande directivité : $\theta_{3 \mathrm{~dB}}=0,6^{\circ}$. L'amplificateur de puissance qui l'ali-

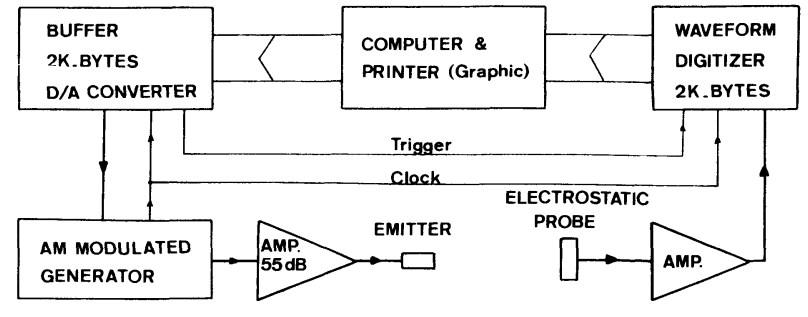

Fig. 2. - Schéma synoptique de l'expérience.

[Schematic of experiment.]

mente permet d'élever la pression émise $P_{0}$ jusqu'à un niveau de $140 \mathrm{~dB} / 1 \mu \mathrm{B}$ ce qui correspond à des valeurs du paramètre $\Gamma$ de Goldberg rapport de la longueur d'atténuation $L_{\mathrm{A}}=\alpha_{0}^{-1}$ (environ $1 \mathrm{~m}$ dans notre expérience) à la longueur de discontinuité calculée pour une onde plane, $L_{\mathrm{D}}=\rho_{0} C_{0}^{3} / 2 \beta \pi v_{-} P_{0}$, pouvant aller jusqu'à 40 ce qui correspond à des niveaux très forts dans le langage défini plus haut. La figure $3 \mathrm{~b}$ montre le résultat obtenu sur l'axe pour une modulation selon une gaussienne (Fig. 3a)

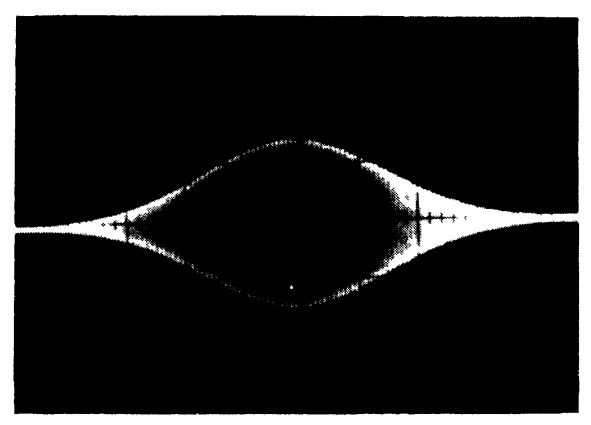

a)

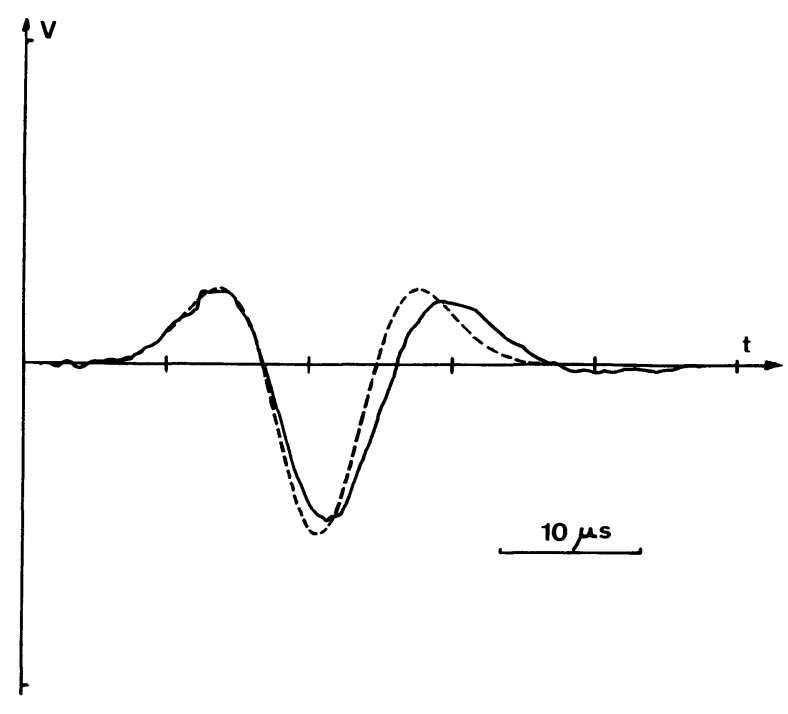

b)

Fig. 3. - Démodulation d'un faisceau modulé par une gaussienne : a) signal primaire; b) signal paramétrique.

[Demodulation of a gaussian modulated beam : a) primary signal; b) parametric signal.] 
comparé au résultat théorique, obtenu à partir de la formule (48) la différence constatée s'explique mieux à l'aide du résultat obtenu à la figure $4 \mathrm{~b}$ associé à une modulation à variation linéaire de fréquence de 0 à $470 \mathrm{kHz}$ (Fig. 4a) lequel manifeste bien une réponse à enveloppe parabolique pour les basses fréquences mais présente un phénomène de saturation à partir de $220 \mathrm{kHz}$ qui reste pour le moment inexplicable. En raison de la grande directivité du faisceau primaire la fonction de directivité $S(\theta, v)$ obtenue pour des régimes paramétriques (Fig. 5) n'est pas très éloignée de l'expression de Westervelt (49).

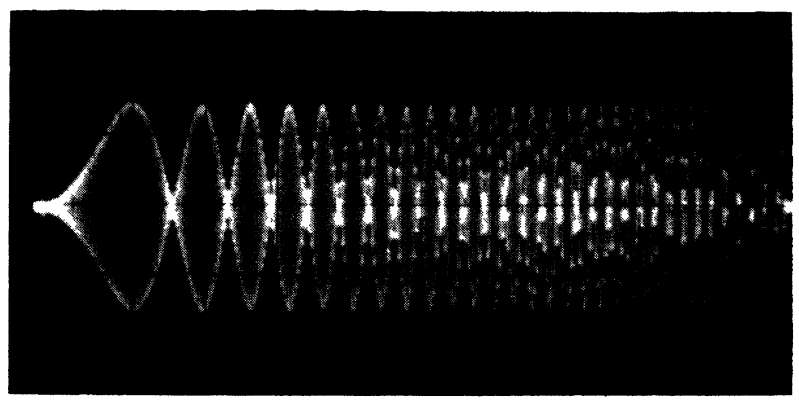

a)

Fig. 4. - Démodulation d'un faisceau modulé par une enveloppe à variation linéaire de fréquence : a) signal primaire; b) signal paramétrique ( $120 \mathrm{~dB}$ réf. $1 \mu \mathrm{B}$ à l'émission); c) signal paramétrique (130 $\mathrm{dB}$ réf. $1 \mu \mathrm{B}$ à l'émission).

[Demodulation of a chirp modulated beam : a) primary signal; b) parametric signal (120 dB ref. $1 \mu \mathrm{B}$ source); c) parametric signal (130 dB ref. $1 \mu \mathrm{B}$ source).]

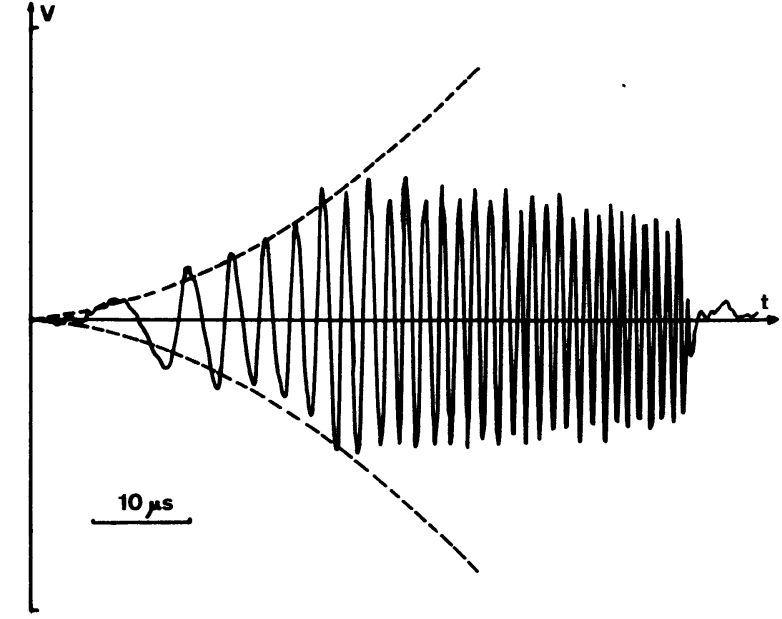

b)

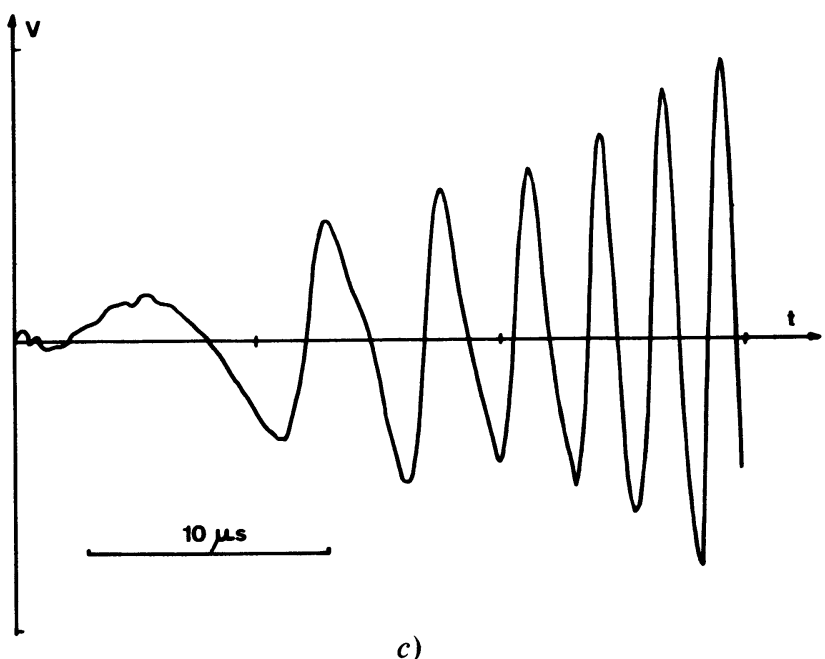

c)

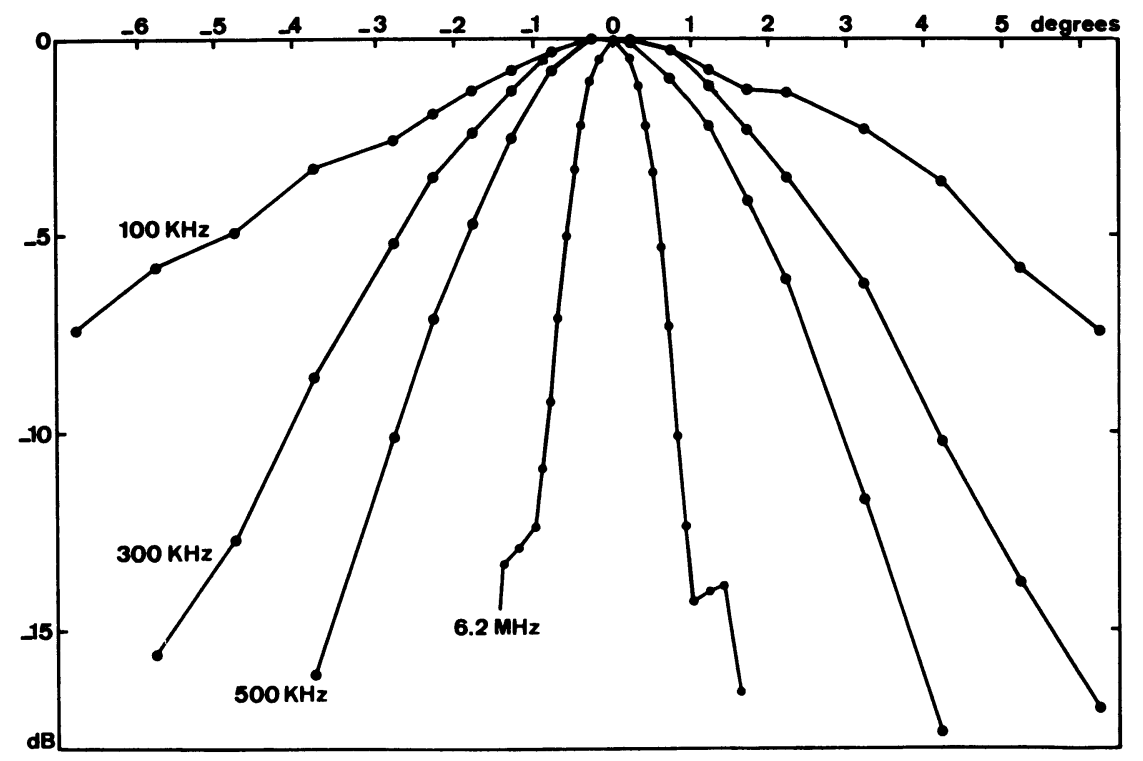

Fig. 5. - Directivités de l'émission primaire $(6,2 \mathrm{MHz})$ et du rayonnement paramétrique pour différentes fréquences.

[Normalized primary $(6.2 \mathrm{MHz})$ and parametric directivities.] 
Un résultat intéressant de ces expériences est que la saturation constatée aux niveaux forts $(\Gamma>1)$ se traduit sur l'axe par une atténuation globale ne modifiant pas sensiblement la forme des signaux. Le chapitre suivant fournit à cela une tentative d'explication.

\section{Extension du modèle aux niveaux forts.}

Aux niveaux forts de puissance, l'énergie cédée par le rayonnement primaire à d'autres fréquences par couplage non linéaire n'est plus négligeable et doit être traduite par une extra-atténuation non linéaire. Comme nous l'avons fait dans une étude antérieure [27], cette situation peut être décrite à l'aide d'une équation modèle, analogue à celle proposée par Kuznetsov [28] :

$$
\Phi=S(\Phi)
$$

où $\square$ ' et $S$ sont les opérateurs décrits dans les équations (1) et (2). Implicitement l'opérateur quadra- tique $S(\Phi)$ ne retient que les couplages binaires de modes connus dans le modèle précédent mais à la différence de ce dernier il n'y a plus hiérarchisation des modes primaires et secondaires. Par exemple si on étudie la propagation d'un rayonnement initialement harmonique de fréquence $v$, le rayonnement apparaîtra comme la somme du fondamental et de ses harmoniques :

$$
\Phi=\sum \Phi_{n}, \quad \Phi_{n}=U_{n}(\mathbf{r}) \mathrm{e}^{-j 2 \pi n v t} .
$$

Sans aucune hiérarchie des $U_{n}$, le mode $U_{2}$ pouvant par exemple au cours de la propagation dominer le mode $U_{1}$ du fait de l'énergie prélevée sur ce dernier. Si on se tient provisoirement à ce cas particulier on peut toujours effectuer une analyse de Fourier du rayonnement dans tout plan $\Pi_{z}(z=\mathrm{Cte})$ discrète en $t(n v)$ et continue spatialement $(\mathbf{m} \rightarrow \mathbf{f})$. L'approximation parabolique décrite plus haut conduit alors aux équations d'évolution des spectres $A_{n}(f, z)$ associés à chaque harmonique $n$ dans le plan $\Pi_{z}$ :

$$
\begin{aligned}
& \begin{aligned}
\frac{\mathrm{d}}{\mathrm{d} z} A_{n}(\mathbf{f}, z)=-\frac{\alpha n^{2}}{\cos \theta_{n}} A_{n}+\frac{2 n^{2} v^{2}}{C_{0}^{3} \cos \theta_{n}} & {\left[\frac{1}{2} \sum_{\substack{p<n \\
n=p+m}} m p \int \beta\left(\boldsymbol{\theta}_{m}, \boldsymbol{\theta}_{p}\right) A_{m}\left(\mathbf{f}^{\prime}, z\right) A_{p}\left(\mathbf{f}-\mathbf{f}^{\prime}, z\right) \mathrm{e}^{j \Delta k_{m p} z} \mathrm{d \mathbf {f } ^ { \prime }}-\right.} \\
& \left.-\sum_{\substack{p^{\prime}>n \\
n=p^{\prime}-m^{\prime}}} m^{\prime} p^{\prime} \int \beta\left(\boldsymbol{\theta}_{m^{\prime}}, \boldsymbol{\theta}_{p^{\prime}}\right) A_{m^{\prime}}^{*}\left(\mathbf{f}^{\prime}, z\right) A_{p^{\prime}}\left(\mathbf{f}^{\prime}+\mathbf{f}, z\right) \mathrm{e}^{j \Delta k_{m^{\prime} p^{\prime} z} \mathrm{~d} \mathbf{f}^{\prime}}\right] \\
& \beta\left(\boldsymbol{\theta}_{m}, \boldsymbol{\theta}_{p}\right)=\mathbf{n}_{m} \cdot \mathbf{n}_{p}+\frac{B}{2 A}
\end{aligned} \\
& \text { où }
\end{aligned}
$$

et $\Delta k_{m p}, \Delta k_{m^{\prime} p^{\prime}}$, représentent le défaut de fermeture (selon $\mathrm{O} z$ exclusivement) des triangles des vecteurs d'ondes : $\mathbf{k}_{m}+\mathbf{k}_{p}$ et $\mathbf{k}_{p^{\prime}}-\mathbf{k}_{m^{\prime}}$. Outre l'approximation paraxiale, $\cos \theta_{n} \cong 1, \beta \cong 1+\frac{B}{2 A}$ l'étude déjà signalée a montré qu'il était possible dans le cas d'un émetteur plan de négliger les rotations de phase en $\Delta k . z$ dans la zone complémentaire de la zone de Fraunhofer précisément celle où l'atténuation linéaire a lieu de façon prédominante. On peut alors dans le cas d'un transducteur plan à centre de symétrie défini par la fonction pupille $\mathcal{S}(\mathbf{p}) \rightleftarrows \mathcal{A}(\mathbf{f})$ proposer une solution à variables séparées pour les spectres $A_{n}$ :

$$
A_{n}(\mathbf{f}, z)=\frac{\lambda}{2 \pi n} U_{n}(z) \mathcal{A}(\mathbf{f}) .
$$

où $U_{n}$ a les dimensions d'une vitesse.

En effet les intégrales dans l'espace de Fourier des $f^{\prime}$ qui apparaissent dans les équations (52) se réduisent pour une telle solution à une autoconvolution du spectre $\mathcal{A}(\mathbf{f})$ qui laisse cette fonction invariante puisque $\mathfrak{T}^{2}(\mathbf{p})=\mathscr{T}(\mathbf{P})$.

La formulation proposée en (53) a l'intérêt de ramener l'étude de la propagation tridimensionnelle du rayonnement $\Phi$ à une étude unidimensionnelle. L'étude [27] a montré que la détermination des $U_{n}(z)$ revenait en fait à une analyse de Fourier de l'équation de Burgers pour un rayonnement initialement harmonique. En effet si on introduit une vitesse de référence $U_{0}, l_{\mathrm{D}}=C_{0}^{2} / 2 \pi \beta \nu U_{0}$ la longueur de discontinuité associée, $\Gamma=\left(\alpha L_{\mathrm{D}}\right)^{-1}$ le paramètre de Goldberg, la notation réduite :

$$
u=U_{n} / U_{0}, \quad \zeta=z / l_{\mathrm{D}}
$$

permet d'écrire les équations vérifiées par les $u_{n}$ selon :

$$
\frac{\mathrm{d} u_{n}}{\mathrm{~d} \zeta}=-\frac{n^{2}}{\Gamma} u_{n}+\frac{n}{2}\left[\frac{1}{2} \sum_{\substack{p<n \\ n=m+p}} u_{m} u_{p}-\sum_{\substack{p^{\prime}>n \\ n=p^{\prime}-m^{\prime}}} u_{m^{\prime}} u_{p^{\prime}}\right]
$$

lesquelles apparaissent bien comme les équations dérivant d'une analyse de Fourier de l'équation modèle de Burgers :

$$
\frac{\partial u}{\partial \zeta}=\frac{1}{\Gamma} \frac{\partial^{2} u}{\partial \zeta}+u \frac{\partial u}{\partial \eta}, \quad \eta=2 \pi v(t-z / c) .
$$

L'intérêt de l'approche ainsi proposée est de fournir 
une ouverture pour le calcul de l'émission paramétrique aux niveaux forts où l'atténuation non linéaire est importante. En effet une analyse de Fourier par les équations (55) du régime paramétrique avec les fréquences primaires $v_{10}$ et $v_{11}$ [27] permet d'atteindre les spectres primaires $A_{10}(f, z)$ et $A_{11}(f, z)$ et d'écrire la création de la fréquence $v_{01}=v_{11}-v_{10}$ paramétrique sous la forme modifiée de l'équation (26) :

$$
\begin{aligned}
\frac{\mathrm{d} A_{-}}{\mathrm{d} z}(\mathbf{f}, z)=-\frac{\beta}{2 C_{0}} & U_{10}^{*}(z) U_{11}(z) \times \\
& \times \int \mathcal{A}^{*}\left(\mathbf{f}_{1}\right) \mathcal{A}\left(\mathbf{f}_{1}+\mathbf{f}\right) \mathrm{e}^{-v z} \mathrm{df}_{1},
\end{aligned}
$$

négligeant l'atténuation faible de la fréquence paramétrique. On peut donc atteindre le spectre paramétrique du champ lointain pour une expression semblable à la formule (33) en écrivant

$$
A_{-\infty}(\mathbf{f})=-\frac{\beta}{2 C_{0}} \int \mathcal{A}^{*}(\mathbf{f}) \mathcal{A}\left(\mathbf{f}_{1}+\mathbf{f}\right) K\left(\mathbf{f}_{1}, \mathbf{f}_{1}+\mathbf{f}\right) \mathrm{d} \mathbf{f}_{1},
$$

où

$$
K\left(\mathbf{f}_{1}, \mathbf{f}_{1}+\mathbf{f}\right)+\int_{0}^{\infty} U_{10}^{*}(z) \cdot U_{11}(z) \mathrm{e}^{-v z} \mathrm{~d} z
$$

Naturellement le noyau $K$ dépend du régime d'excitation et doit être recalculé pour tout niveau de puissance considéré, à partir de la solution correspondante $U(z)$ de l'équation de Burgers.

$\mathrm{Ce}$ formalisme a ainsi été mis en œuvre pour reconstruire l'évolution d'une onde sinusoïdale pure à l'émission, en fonction du paramètre $\Gamma$ [29] ainsi que l'évaluation du diagramme de rayonnement secondaire d'un sonar paramétrique rectangulaire plan [30], les résultats offrant une très bonne concordance avec les relevés expérimentaux correspondants (Figs. 6a et $b$ ).

On peut aussi proposer une extension aux niveaux forts de puissance de la théorie de l'autodémodulation non linéaire proposée au chapitre 4 . En effet si on admet que les effets non linéaires sur le rayonnement primaire se traduisent par une atténuation globale du spectre temporel $r\left(v-v_{0}\right)$ introduit en (42), conservant la forme de ce dernier, on peut admettre une expression du spectre primaire :

$$
A(\mathbf{f}, v, z)=\mathcal{A}(\mathbf{f}) \quad r\left(v-v_{0}\right) u(z),
$$

où $u(z)$ est solution de l'équation de Burgers pour la fréquence centrale $v_{0}$. Conservant les notations du $\S 4$ on peut alors proposer la solution en champ lointain pour le champ basse fréquence résultant de l'autodémodulation :

$$
\begin{gathered}
A_{-}(\mathbf{f}, v)=G(v) . S^{\prime}(v, \boldsymbol{\theta}), \\
\operatorname{avec} S^{\prime}(v, \boldsymbol{\theta})=\int^{*} \mathcal{A}^{*}\left(\mathbf{f}_{1}\right) \mathcal{A}\left(\mathbf{f}_{1}+\mathbf{f}\right) K\left(\mathbf{f}_{1}, \mathbf{f}\right) \mathrm{d} \mathbf{f}_{1}, \\
K\left(\mathbf{f}_{1}, \mathbf{f}\right)=2 \alpha_{0} \int_{0}^{\infty} u^{*}(z) u(z) \mathrm{e}^{j \pi \gamma\left(\mathbf{f}_{1}-n \mathbf{f}\right)^{2 z} z} \mathrm{~d} z, \\
\gamma=c v / 2 \alpha_{0} v_{0}^{2} .
\end{gathered}
$$
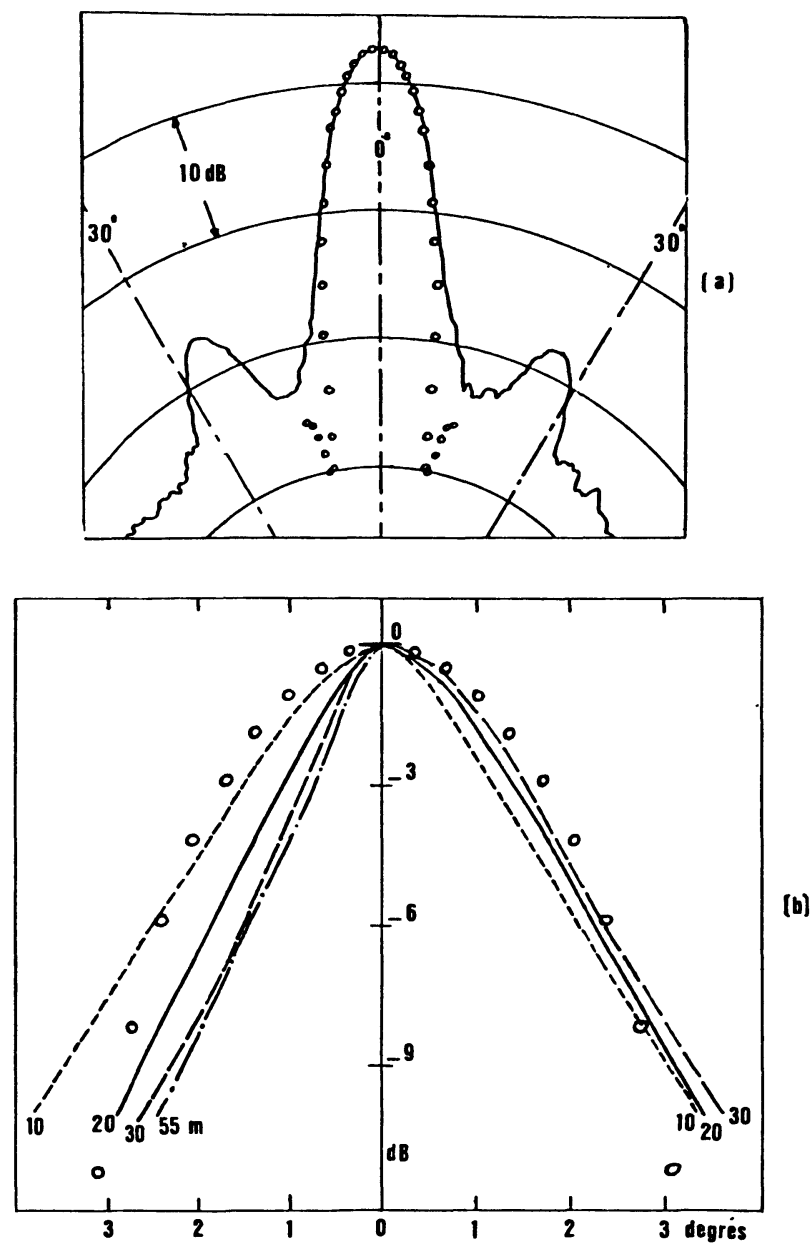

Fig. 6. - Diagrammes de rayonnement secondaire en site (a) et en gisement (b) d'un émetteur rectangulaire plan, dans l'eau de mer. Taille de l'émetteur : $30 \mathrm{~cm} \times 4,8 \mathrm{~cm}$; fréquence médiane $\mathrm{HF}: 200 \mathrm{kHz}$; fréquence paramétrique : $20 \mathrm{kHz}$. Niveau dé source (pour $1 \mathrm{Hf}$ ) : $115 \mathrm{~dB}$ réf. $1 \mu \mathrm{B}$ à $1 \mathrm{~m}$; 000 : valeurs calculées, en champ lointain; - _ relevés expérimentaux.

[Secondary beam patterns in site (a) and bearing (b) of a plane rectangular source, in sea water. Emitter size : $30 \mathrm{~cm} \times$ $4,8 \mathrm{~cm}$; middle high frequency : $200 \mathrm{kHz}$; parametric frequency : $20 \mathrm{kHz}$; source level : $115 \mathrm{~dB}$ ref. $1 \mu \mathrm{B}$ at $1 \mathrm{~m}$; o o o : farfield computed values; _ measures.]

Cette dernière formulation permet d'expliquer que dans le cas des transducteurs directifs pour lesquels les composantes spectrales vérifient pour l'essentiel : $\gamma f_{1}^{2} \ll 1$, la solution sur l'axe $A_{-}(0, v)$ garde la même forme que pour les niveaux faibles, ce qui est expérimentalement très bien constaté.

Par ailleurs, la remarque concernant la faible valeur de la désadaptation en phase $\delta \varphi=\Delta k_{z} z \ll 1$ des modes participant à la création des harmoniques dans le cas d'une excitation harmonique pure peut s'étendre au cas considéré ici d'une excitation à bande étroite et permet d'écrire le spectre harmonique II relié sur la fréquence $2 v_{0}$ :

$$
A^{\mathrm{II}}\left(\mathbf{f}, v-2 v_{0}, z\right)=F\left(v-2 v_{0}\right) \mathcal{A}(\mathbf{f}) 2 \alpha_{0} u^{\mathrm{II}}(z),
$$


où $u^{\text {III }}(z)$ est la solution de l'équation de Burgers à une excitation harmonique de fréquence $v_{0}$ pour l'harmonique II de fréquence $2 v_{0}$. Si on admet que l'essentiel de l'énergie transférée aux fréquences $2 v_{0}$ provient de l'addition des fréquences primaires centrées sur $v_{0}$ la fonction $F$ s'identifie à la fonction $G$ autoconvolution de la fonction de modulation. Aux niveaux élevés d'excitation l'autodémodulation non linéaire associée au rayonnement $A^{\text {II }}$ s'ajoute au rayonnement $A_{-}$décrit précédemment et provoque une distorsion sensible du signal qu'on peut apprécier sur la figure $4 \mathrm{c}$ laquelle fournit le résultat obtenu pour la modulation décrite à la figure $4 \mathrm{a}$ mais pour une pression émise $P_{0}$ de $130 \mathrm{~dB}(\Gamma \cong 13)$.

\section{Conclusion.}

Nous avons pu montrer dans ce texte qu'on peut atteindre correctement par une analyse de Fourier en modes plans atténués en $z$ la propagation linéaire dans un fluide en prenant l'atténuation en compte ainsi que le développement des phénomènes non linéaires à des niveaux d'excitation ne provoquant pas d'atténuation non linéaire sensible sur le rayonnement primaire. Les cas particuliers de l'émission paramétrique et de l'autodémodulation non linéaire lesquels sont susceptibles d'applications pratiques pour la prospection géologique, la détection sousmarine ou les télécommunications sous-marines ont été particulièrement détaillés.

Enfin, nous avons montré également que la restriction aux niveaux faibles peut être levée par une équation modèle toujours limitée à des interactions binaires de modes mais prenant en compte l'évolution non linéaire du rayonnement primaire. Dans le cas de transducteurs assez directifs, l'analyse unidimensionnelle résultant de l'équation de Burgers peut être exploitée pour accéder au rayonnement tridimensionnel de basse fréquence.

\section{Bibliographie}

[1] Goldberg, Z. A., Second approximation acoustic equations and the propagation of plane wave of finite amplitude. Sov. Phys. Acoust. 2 (1956) 346.

[2] Westervelt, P. J., Scattering of sound by sound. JASA 29, $\mathrm{n}^{\circ} 2$ (1957) 199-203.

[3] Westervelt, P. J., Parametric acoustic array, JASA $35, n^{\circ} 4$ (1963) 535-537.

[4] Berktay, H. O. and Leahy, D. J., Farfield performance of parametric transmitters, JASA 55, $\mathrm{n}^{\circ} 3$ (1974) 539-546.

[5] Moffett, M. B. and Mellen, R. H., Model for parametric acoustic sources, JASA 61, $\mathrm{n}^{\mathrm{o}} 2$ (1977) 325-337.

[6] Moffett, M. B. and Mellen, R. H., A model for parametric acoustic sources, Naval underwater systems center report, New London, Connecticut (1976).

[7] Hennion, P. Y., Les interactions non linéaires d'ondes acoustiques. Etude par une méthode de Fourier. Applications à l'émission paramétrique. Thèse de Doctorat d'Etat ès Sciences Physiques, Paris VI (1984).

[8] LAUNSTAD, V. R., Non linear interaction of two monochromatic sound waves, Acustica 16 (1965) 191-207.

[9] BLACKSTOCK, D. T., Propagation of plane sound waves of finite amplitude in non dissipative fluids, JASA 34, $\mathrm{n}^{\circ} 1$ (1962) 9-30.

[10] Blackstock, D. T., Thermoviscous attenuation of plane, periodic, finite-amplitude sound waves, JASA 36, $\mathrm{n}^{\circ} 3$ (1964) 534-542.

[11] Alais, P., Effets de l'atténuation sur un rayonnement quelconque dans un milieu propagatif linéaire absorbant, C. R. Hebd. Séan. Acad. Sc. Paris, Série A, 282 (1976) 547.
[12] Goodman, J. W., Introduction à l'optique de Fourier et à l'holographie (Masson, Paris) (1972).

[13] Alais, P. et Hennion, P. Y., Interaction non linéaire paramétrique de deux modes acoustiques d'ondes planes atténuées inhomogènes, C. R. Hebd. Séan. Acad. Sc. Paris, Série B, 282, $\mathrm{n}^{\circ} 17$ (1976) 385387.

[14] Alais, P. et Hennion, P. Y., Etude par une méthode de Fourier de l'interaction non linéaire de deux rayonnements acoustiques dans un fluide absorbant. Cas particulier de l'émission paramétrique. Acustica 43, $\mathrm{n}^{\mathrm{o}} 1$ (1979) 1-11.

[15] Alais, P. et Hennion, P. Y., Interaction non linéaire dans un milieu absorbant de deux rayonnements acoustiques monochromatiques. C. R. Hebd. Séan. Acad. Sc. Paris, Série B 282 (1976) 421-423.

[16] Hennion, P. Y. et Alais, P., Etude du rayonnement paramétrique de transducteurs focalisants. Revue du CETHEDEC 56 (1978) 17-21.

[17] Cervenka, P., Analyse de Fourier du rayonnement d'émetteurs paramétriques. Thèse de $3 \mathrm{e}$ cycle, Paris VI, Phys. (1982).

[18] Berktay, H. O., Possible exploitation of non linear acoustics in underwater transmitting applications. JSV 2, no 4 (1965) 435-461.

[19] BLACKsTOCK, D. T., Transient solution for sound radiated into a viscous fluid. JASA 41, $\mathrm{n}^{\circ} 5$ (1967) 1312-1319.

[20] Moffett, M. B. and BeYER, R. T., Transient effects in the propagation of a sound pulse in a viscous liquid. JASA 47, $\mathrm{n}^{\mathrm{o}} 5$ (1970) 1241-1249.

[21] Moffett, M. B., Westervelt, P. J. and Beyer, R. T., Large-amplitude pulse propagation. A transient effect. JASA 47, no 5 (1970) 1473-1474. 
[22] Moffett, M. B., Westervelt, P. J. and Beyer, R. T., Large-amplitude pulse propagation. A transient effect. II. JASA 49, $\mathrm{n}^{\circ} 1$ (1971) 339-343.

[23] Moffett, M. B. and Mello, P., Parametric acoustic sources of transient signals. JASA 66, $\mathrm{n}^{\circ} 4$ (1979) 1182-1187.

[24] Merklinger, H. M., Improved efficiency in the parametric transmitting array. JASA 58 no 4 (1975) p. 784-787.

[25] Grinberg, I. E., Novikov, B. K. and Timoshenko, V. I., Parametric array in the self-demodulation regime. Sov. Phys. Acoust. 30 n $^{\circ} 2$ (1984) p. 118-120.

[26] Alais, P. and Cervenka, P., Theoretical and experimental study of the parametric self demodulation, 13th ISNA (KOBE/07/84).
[27] Alais, P., Hennion, P. Y. et Lagreve, M., Théorie de Fourier de la propagation linéaire et non linéaire dans un fluide absorbant. Application à la transduction paramétrique. J. Physique Colloq. 40 no 11 (1979) C8-83-88.

[28] KuZNETSOv, V. P., Equations of nonlinear acoustics. Sov. Phys. Acoust. 16 no $^{\circ}$ (1971) p. 467-470.

[29] Hennion, P. Y. et LaGreve, M., Calcul de l'atténuation non linéaire d'une onde acoustique plane d'amplitude finie. Acustica 48 no $^{\circ}$ (1981) p. 44-49.

[30] Hennion, P. Y. et Cervenka, P., Etude de l'interaction paramétrique de champs ultrasonores d'amplitude finie. Rev. CETHEDEC 71 (1982) p. 13-19. 